\title{
Evaluación del riesgo de contaminación con nitrato de pozos de suministro de agua potable rural en Chile
}

\author{
José Luis Arumi, ${ }^{1}$ Jorge Núñez, ${ }^{2}$ Luis Salgado $^{1}$ y Marcelino Claret $^{3}$
}

Forma de citar Arumi JL, Núñez J, Salgado L, Claret M. Evaluación del riesgo de contaminación con nitrato de pozos de suministro de agua potable rural en Chile. Rev Panam Salud Publica. 2006;20(6):385-92.

RESUMEN Objetivos. Evaluar el riesgo asociado a la contaminación con nitrato de pozos noria de suministro de agua potable rural en la zona de Parral, Chile.

Métodos. Se recogieron datos de concentración de nitrato obtenidos de un muestreo de agua de 94 pozos noria. Se analizó la distribución de la concentración de nitrato en los pozos para determinar la existencia de algún tipo de correlación espacial. En el análisis de riesgo, se identificaron dos situaciones de exposición de la población (adultos y lactantes) y se elaboraron mapas de riesgo para la salud.

Resultados. El $14 \%$ de los pozos estudiados presentó valores de concentración de nitrato mayores que los permitidos por la normativa nacional relativa al agua potable. No se detectó correlación espacial de las concentraciones de nitrato. El valor medio del cociente de peligro (CP) para los adultos en la zona de estudio fue 0,12, lo que indica la ausencia de riesgo para la salud de esa población. Para los lactantes, el cociente de peligro medio fue 0,69 , pero se identificaron algunos pozos donde el cociente de peligro indica un riesgo para esta población.

Conclusiones. En la zona de Parral, la contaminación de pozos noria por nitrato está asociada principalmente a la existencia de ciertos factores, como los métodos constructivos o la cercanía de animales, que afectan de manera aislada la calidad del agua. No se detectó la existencia de riesgo para la población adulta, pero sí para los lactantes alimentados con fórmulas preparadas con agua proveniente de los pozos contaminados.

Palabras clave Medición de riesgo, pozos, nitratos, agua potable, población rural, Chile.

La contaminación de las aguas subterráneas es un problema creciente de

\footnotetext{
Universidad de Concepción, Departamento de Recursos Hídricos, Chillán, Chile. Toda correspondencia deberá dirigirse a: Dr. José Arumi, Universidad de Concepción, Departamento de Recursos Hídricos, Vicente Méndez 595, Chillán, VIII Región, Chile; teléfono: (56) 42 208804; fax: (56) 42 275303; correo electrónico: jarumi@udec.cl

Ingeniero consultor

3 Instituto de Investigaciones Agropecuarias, Departamento de Recursos Naturales y Medio Ambiente, Ministerio de Agricultura, Chile
}

la salud pública, pues restringe la disponibilidad de agua potable para la población. En Chile, el agua subterránea para consumo humano representa más del $40 \%$ del volumen total de agua consumido en las zonas urbanas y el $76 \%$ en las zonas rurales (1).

El nitrato es uno de los contaminantes mas frecuentes de las aguas subterráneas y su presencia puede asociarse a problemas de salud humana, especialmente en grupos sensibles como los lactantes. Estos, al ingerir fórmulas lácteas preparadas con agua con altas concentraciones de nitrato, pueden presentar metahemoglobinemia $\mathrm{O}$ «síndrome del niño azul» $(2,3)$. A pesar de que esto ha sido recientemente cuestionado (4), las normativas relativas a la calidad del agua y los métodos de análisis de riesgo consideran esta hipótesis dentro de su fundamentación, pues están concebidos para proteger la salud humana $(2,3)$. 
En Chile, los procesos de contaminación asociados a sistemas de producción agrícola han comenzado a estudiarse recientemente. Afortunadamente, se han encontrado pruebas de que en el valle central, existen condiciones naturales generalizadas de protección de los sistemas de aguas subterráneas frente a contaminaciones por nitrato asociadas a prácticas de fertilización (5). Sin embargo, se han detectado casos de contaminación por mal manejo de las aguas residuales y los desechos agrícolas (6)

En este contexto, a pesar de las grandes mejoras realizadas en materia de abastecimiento de agua potable de la población rural, existen aún zonas donde los pobladores se aprovisionan de agua a través de pozos noria que pueden presentar problemas de contaminación y donde el contenido de nitrato sobrepasa los límites de las normas nacional e internacionales, lo que conlleva una preocupación por el efecto que puedan provocar esas concentraciones en la población (7). La norma chilena establece un límite máximo de nitrato, expresado como cantidad de nitrógeno en forma de nitrato $\left(\mathrm{N}-\mathrm{NO}_{3}{ }^{-}\right)$, de $10 \mathrm{mg} / \mathrm{L}$, equivalente al valor fijado por la Agencia de Protección Ambiental de los Estados Unidos (USEPA) y recomendado por la Organización Mundial de Salud (OMS) (2).

Como el costo de aplicación de procesos de recuperación de lugares contaminados excede la disponibilidad de financiamiento, se han desarrollado metodologías, como la evaluación de riesgos, para seleccionar aquellos lugares que implican un mayor riesgo para la salud pública. (8).

En el presente trabajo se utiliza la metodología recomendada por la USEPA para evaluar el riesgo para la salud de una parte de la población rural expuesta a aguas contaminadas con nitrato en la zona de influencia del Embalse Digua, Parral, Séptima Región de Chile (9).

\section{MATERIALES Y MÉTODOS}

La zona de estudio tiene una superficie aproximada de 65700 ha y se en- cuentra entre las coordenadas $36^{\circ} 12^{\prime}$ de latitud sur y $72^{\circ}$ de longitud oeste. En esta zona hay aproximadamente 1200 agricultores, en su mayoría usuarios del embalse Digua (7).

La información básica para este estudio estuvo constituida por los valores de concentración de nitrato, expresados en $\mathrm{mg} / \mathrm{L}$ de $\mathrm{N}-\mathrm{NO}_{3}{ }_{3}$, obtenidos por el Instituto de Investigaciones Agropecuarias (7) en muestras de agua tomadas en 92 pozos noria ubicados en la zona de estudio (figura 1). Como es usual que cada casa rural tenga un pozo, se puede estimar que el número total de pozos existentes en la zona de estudio es aproximadamente 1200.

El diseño de muestreo elegido fue un modelo sistemático no alineado, donde dentro de cada cuadrante de la malla resultante se obtuvo un punto al azar. Utilizando la plataforma SIG Map-Info, la malla de muestreo se sobrepuso a la cobertura predial previamente digitalizada y georreferenciada con un receptor de GPS III ${ }^{\circledR}$ Plus, de Garmin. El análisis de nitrato se realizó por el método de reducción con cinc en granallas (malla 20) (7).

El análisis geoestadístico se efectuó con el programa Variowin ${ }^{\circledR}$, el módulo geoestadístico de Surfer ${ }^{\circledR} 7$ y el paquete GS $+{ }^{\circledR}$. El análisis estadístico descriptivo se realizó con el programa Statgraphics Plus ${ }^{\circledR}$. Para elaborar los mapas de riesgo se utilizó el programa Surfer ${ }^{\circledR}$ 7. El análisis de incertidumbre se realizó con el programa Simlab ${ }^{\circledR}$ y el complemento de Excel ${ }^{\circledR}$ Analyze-It ${ }^{\circledR}$.

\section{Análisis de los datos}

La etapa inicial consistió en un análisis estadístico descriptivo. Este análisis permitió caracterizar la concentración de nitrato respecto a medidas de tendencia central y a la varianza. Las curvas de frecuencia, asociadas con diversas pruebas de normalidad, indican el tipo de distribución al que se ajustan los datos.

Para el análisis geostadístico, se buscó identificar entre distintos métodos de interpolación, el más apropiado según las características de continuidad espacial de los datos: métodos de Kriging, de ponderación en función inversa de la distancia elevada a una potencia, del vecino más cercano, del vecino natural, etc. (10).

La segunda etapa consistió en la identificación de los parámetros toxicológicos del nitrato utilizados en la caracterización del riesgo (compuesto inorgánico no cancerígeno, dosis de referencia [DdR] recibida por vía oral en agua: $1,6 \mathrm{mg} \mathrm{kg}^{-1}$ día $\left.^{-1}\right)$. El parámetro utilizado fue la dosis de referencia, que indica el grado de exposición diaria que no implica un riesgo apreciable de daño en poblaciones humanas, incluidas las subpoblaciones sensibles (8).

La tercera etapa consistió en la evaluación de la exposición, que permitió definir la ruta y la vía de exposición. Se supuso que el nitrato tiene su origen en las prácticas agrícolas de fertilización, la eliminación de residuos animales o el tratamiento de aguas servidas. El nitrato lixivia hasta el acuífero, desde donde puede llegar a los pozos que se utilizan como fuente de bebida. En la evaluación se consideraron dos hipótesis: exposición de adultos y exposición de lactantes en los primeros 3 meses de vida, correspondientes al periodo de máxima sensibilidad a la intoxicación por nitrato (2). El cuadro 1 presenta la caracterización de las hipótesis propuestas.

En la cuarta etapa se combinó la información proveniente de los análisis previos para calcular el riesgo, expresado como cociente de peligro $(8,11)$ :

$$
C P=\frac{\left[\frac{C \cdot I \cdot F E \cdot D E}{P C \cdot T P}\right]}{D d R}
$$

Donde CP es el cociente de peligro (adimensional); C, la concentración de nitrato en el agua en cada punto del mapa, en $\mathrm{mg} / \mathrm{L}$; I, la ingesta de agua, en L/día; FE, la frecuencia de exposición, en días/año; DE, la duración de la exposición, en años; PC, el peso corporal, en kg; TP, el tiempo de pro- 
FIGURA 1. Ubicación del área de estudio y de los puntos de muestreo

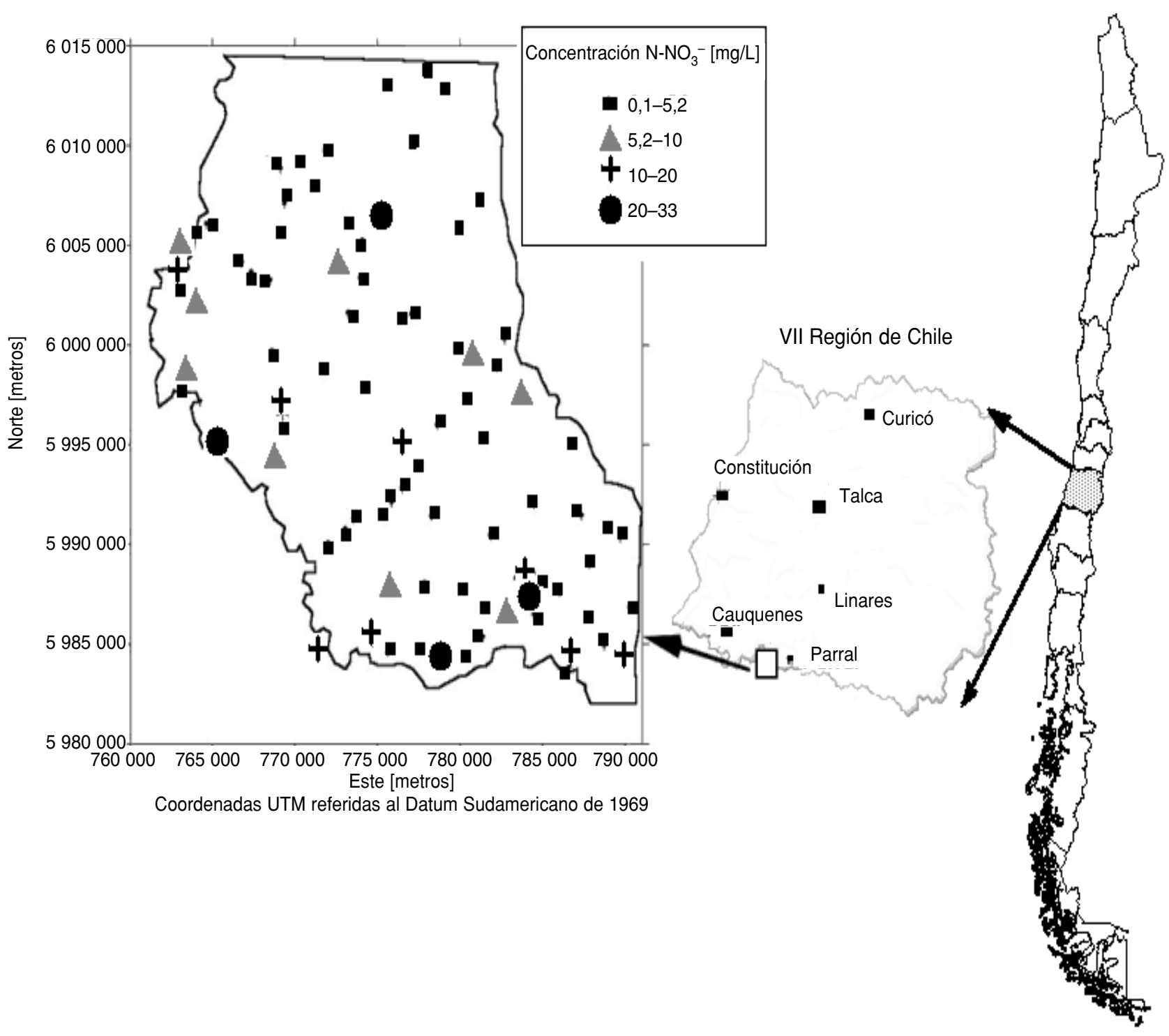

mediación, equivalente a la DE multiplicada por 365; y DdR, la dosis de referencia. La dosis de referencia se obtiene a partir de investigaciones donde se detectan, en forma experimental o estadística, los grados máximos de exposición que no causan daños, aplicando después factores de incertidumbre (8). En el caso del nitrato, la dosis de referencia es $1,6 \mathrm{mg}$ $\mathrm{kg}^{-1}$ día $^{-1}$ (12).

En la etapa final, se consideró el hecho de que el modelo de cálculo de riesgo presenta un grado de incertidumbre en los valores de los parámetros de entrada. De este modo, se identificaron los factores que determinan en mayor grado el valor del cociente de peligro mediante un análisis de sensibilidad, con la ayuda de un diagrama de tornado y el cálculo de los coeficientes de correlación de Spearman (rs) entre cada parámetro y el cociente de peligro. El cuadro 2 presenta los parámetros utilizados en el análisis de sensibilidad.

\section{RESULTADOS}

La figura 2 muestra gráficas de frecuencia y diagramas de cajas y de barras de la concentración de nitrato $\left(\mathrm{N}-\mathrm{NO}_{3}{ }^{-}\right)$en los pozos estudiados. La concentración media es $5,2 \mathrm{mg} / \mathrm{L}$, valor inferior al límite de seguridad recomendado de $10 \mathrm{mg} / \mathrm{L}$ (2). La mediana es $2,6 \mathrm{mg} / \mathrm{L}$, mucho menor que dicho límite. A pesar de que la distribución es asimétrica, la media tiene mayor importancia que la mediana, 
CUADRO 1. Caracterización de las hipótesis de exposición consideradas en este estudio

\begin{tabular}{lrr}
\hline & \multicolumn{2}{c}{ Población } \\
\cline { 2 - 3 } \multicolumn{1}{c}{ Parámetro } & Adultos & Lactantes \\
\hline Peso corporal (kg) & 70 & 4 \\
Tasa de ingesta (L/día) & 2 & 0,64 \\
Duración de la exposición (años) & 30 & 0,25 \\
Frecuencia de la exposición (días/año) & 350 & 350 \\
\hline
\end{tabular}

CUADRO 2. Caracterización de los parámetros utilizados en el análisis de sensibilidad

\begin{tabular}{|c|c|c|c|c|}
\hline Parámetro & Mínimo & Base & Máximo & Fuente \\
\hline Concentración de $\left(\mathrm{N}^{-\mathrm{NO}_{3}}{ }^{-}\right)^{\mathrm{a}}(\mathrm{mg} / \mathrm{L})$ & 0,1 & 30 & 32,5 & (7) \\
\hline Ingesta de agua (L/día) & 0 & 0,64 & 0,80 & (15) \\
\hline Peso corporal $(\mathrm{kg})$ & 3 & 4 & 8 & (15) \\
\hline Frecuencia de la exposición (días/año) & 0 & 350 & 365 & (9) \\
\hline Duración de la exposición & \multicolumn{4}{|c|}{$\begin{array}{l}\text { Teniendo en cuenta que la duración de la exposición no influye } \\
\text { en los cálculos, se considera constante e igual a } 0,25 \text { año. }\end{array}$} \\
\hline
\end{tabular}

FIGURA 2. Gráficas de a) distribución de frecuencia absoluta, b) frecuencia relativa acumulada, c) probabilidad normal y d) caja y barras para las concentraciones de nitrógeno en forma de nitrato $\left(\mathrm{NO}_{3}^{-}\right)$medidas en los pozos noria
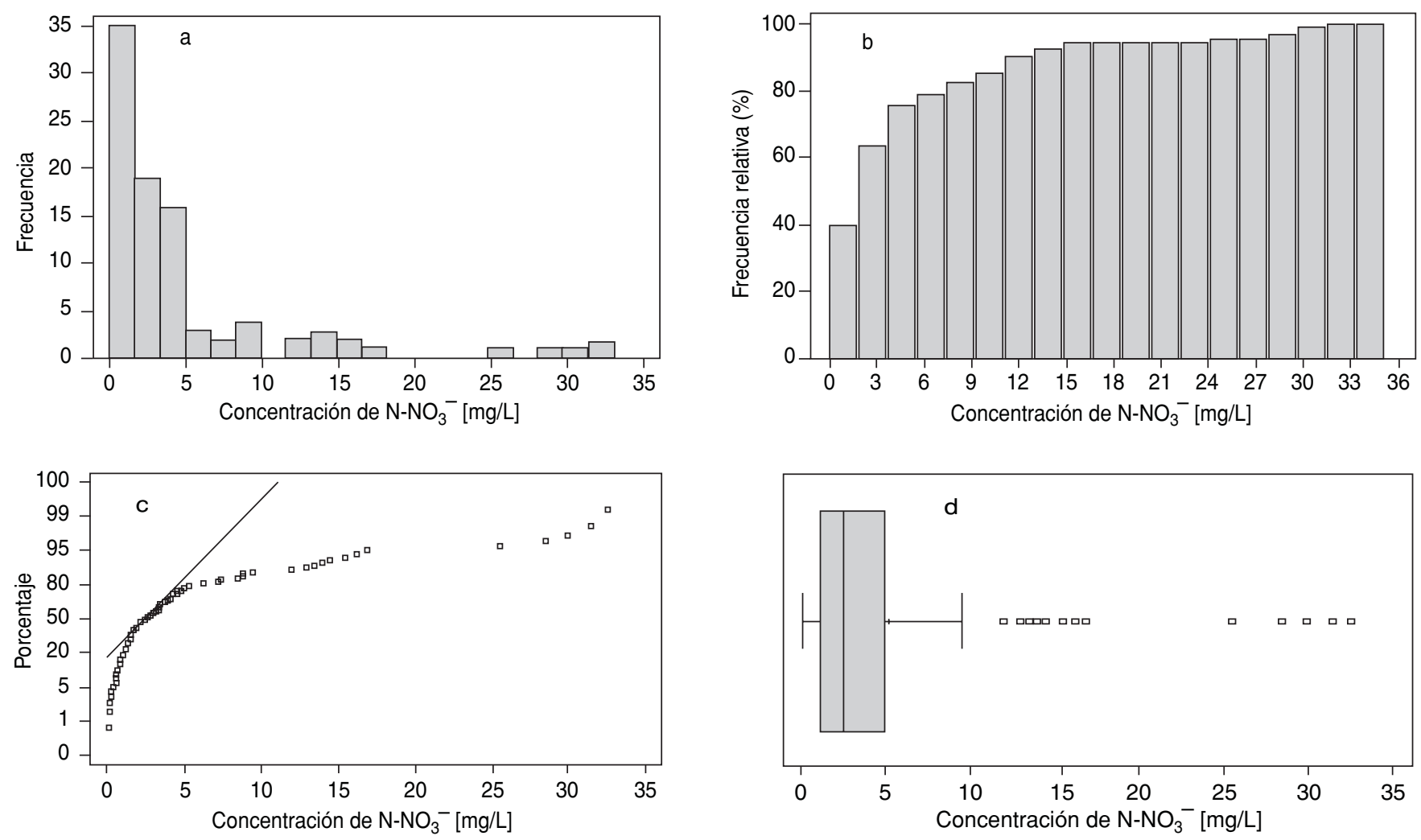
CUADRO 3. Prueba de normalidad respecto de la variable transformada "logaritmo natural de la concentración de nitrógeno en forma de nitrato» $\left[\mathrm{Ln}\left(\mathrm{N}-\mathrm{NO}_{3}^{-}\right)\right]$

\begin{tabular}{lc}
\hline \multicolumn{1}{c}{ Prueba } & $P^{a}$ \\
\hline Anderson y Darling & 0,6446 \\
Sesgo & 0,9855 \\
Apuntamiento (curtosis) & 0,1945 \\
\hline
\end{tabular}

$P>0,1$ indica que no se rechaza la hipótesis de normalidad con un grado de confianza del $90 \%$ o superior.

al considerarla como un parámetro que determina la exposición de la población en un periodo determinado (11). La desviación estándar es de 7,09 mg/L y, en relación con la media, determina un coeficiente de variación del $137,6 \%$, lo que indica una gran variación entre las muestras. El $14 \%$ de las muestras presentó valores mayores que el límite máximo recomendado para el agua de bebida, de $10 \mathrm{mg} / \mathrm{L}$ (5).

La distribución de probabilidad se ajustó al tipo lognormal, verificado por la prueba de normalidad de Anderson y Darling respecto de los datos transformados (ver el cuadro 3). Este tipo de distribución es similar al encontrado por otros autores (13) en muestras de aguas de pozos en zonas agrícolas del sudoeste del Uruguay y es representativo del comportamiento general asociado a variables ambientales especialmente relacionadas con aguas subterráneas (14). Es importante considerar este último aspecto en el análisis geoestadístico para definir el tipo de interpolación que debe utilizarse.

\section{Análisis geoestadístico}

Las variables ambientales distribuidas espacialmente deben presentar un mínimo de correlación espacial, a menos que exista justificación física para establecer la independencia. En este caso, las características constructivas de los puntos de muestreo (pozos noria), totalmente expuestos al ambiente y a la intervención humana, pueden explicar la falta de correlación espacial.
FIGURA 3. Mapa de isolíneas de concentración de nitrógeno en forma de nitrato $\left(\mathrm{N}-\mathrm{NO}_{3}{ }_{3}^{-}, \mathrm{mg} / \mathrm{mL}\right.$ ) detectada en el área de estudio (Parral, Chile, 2002)

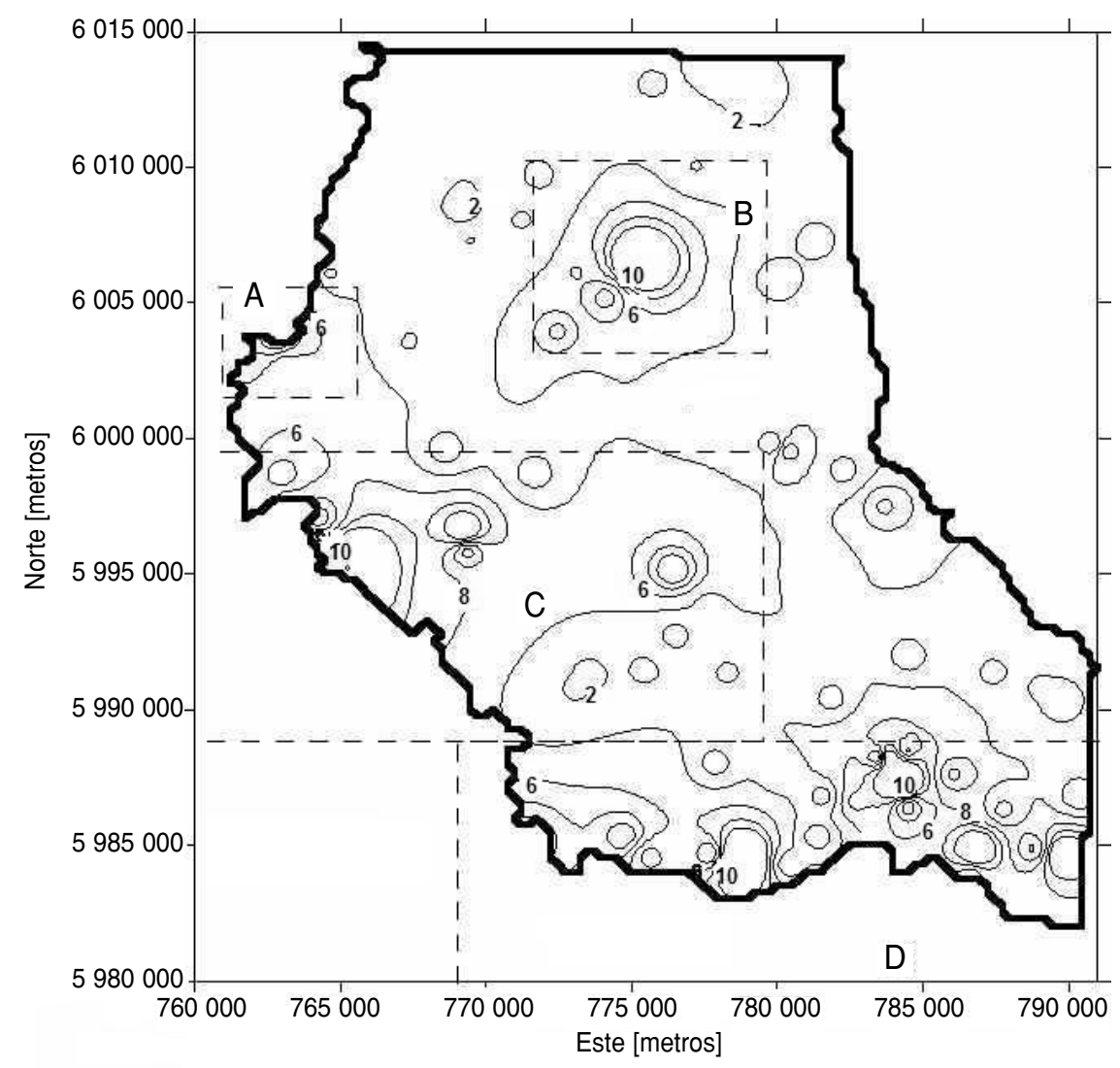

En el análisis geoestadístico no se detectó continuidad espacial de la concentración de nitrato, ni en su valor original ni en el valor transformado logaritmicamente, por lo que no se consideró conveniente utilizar el método de interpolación de Kriging, sino un método más simple, el de de ponderación en función inversa de la distancia elevada a una potencia (10). La figura 3 presenta un mapa de distribución de la concentración de nitrato en la región de estudio, en el que se observa la existencia de cuatro zonas importantes, ya sea por el área de influencia o por la magnitud de la concentración. Las zonas localizadas hacia el límite norte $(\mathrm{A}$ y $\mathrm{B})$ de la región de estudio aparecen más bien como puntos contaminados; en el centro (C) y hacia el límite sur (D), las zonas se encuentran más distribuidas. Lamentablemente, no se contó con información adicional para identificar el origen o los mecanismos de transporte del nitrato en el acuífero.

\section{Caracterización del riesgo}

La figura 4a muestra el mapa de riesgo en la primera hipótesis de exposición. El valor máximo del cociente de peligro fue 0,56 y la media del cociente de peligro de la zona, 0,12. De este modo, no existe riesgo para la población adulta y, al ser el cociente de peligro menor que 1 , no se requiere un análisis de incertidumbre (16).

La figura $4 b$ presenta el mapa de riesgo para la salud en la segunda hipótesis de exposición, caracterizada por la presencia de un grupo de entre 0 y 3 meses de edad, considerado como el más sensible a la intoxicación por nitrato $(2,3)$. En la zona de estudio, el cociente de peligro alcanzó un 
FIGURA 4. Mapa de isolíneas de cociente de peligro (CP) para la salud de a) adultos y b) lactantes, en el área de estudio (Parral, Chile, 2002). (En la figura $4 b$ se han resaltado las isolíneas correspondientes a $C P=1,0$ )
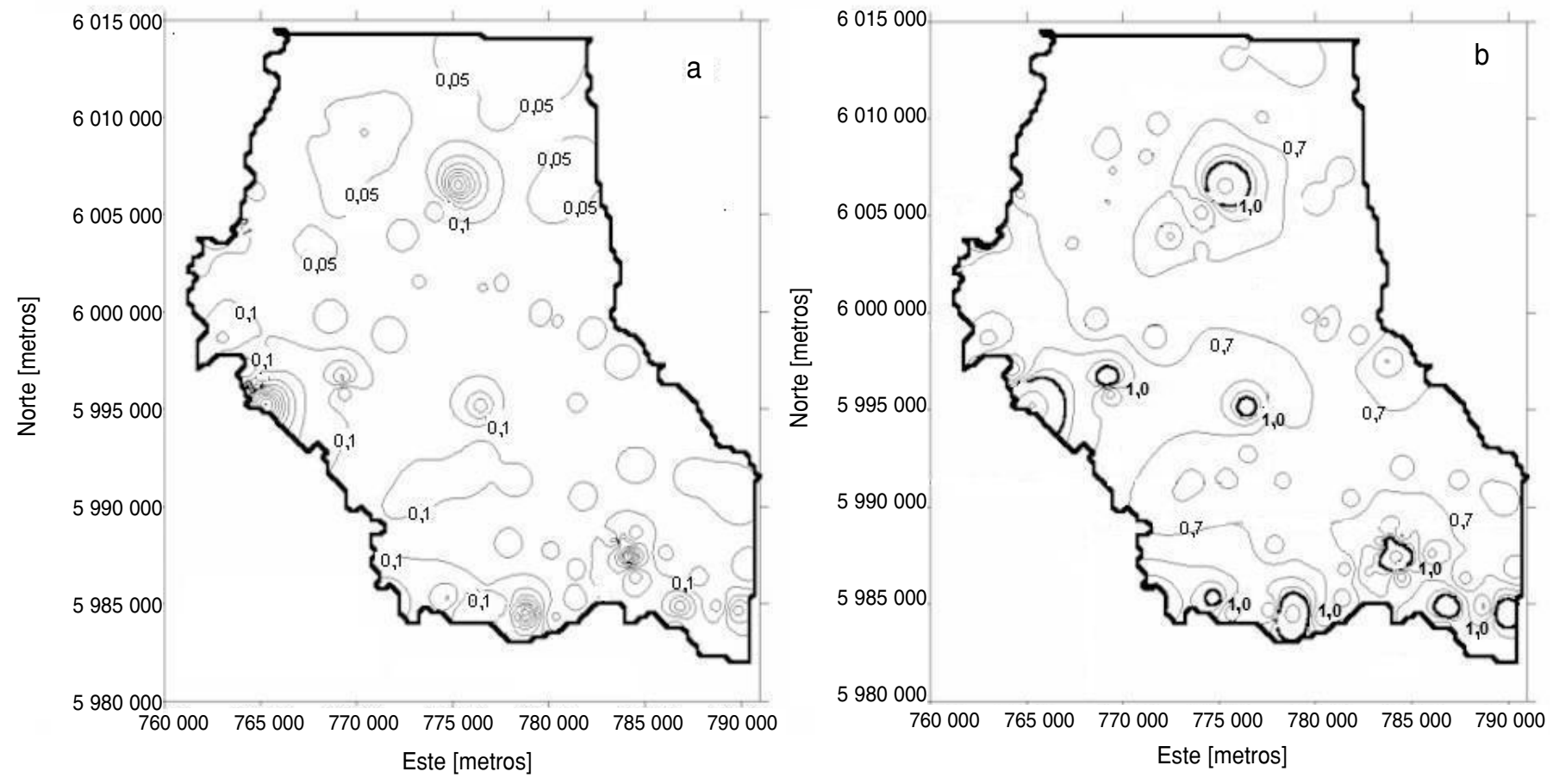

valor máximo de 3,1 en algunos puntos, pero el valor medio fue de 0,69 . Esto indica que aunque existen algunos pozos de los cuales es riesgoso obtener agua para lactantes, ésta no es la tendencia general de la zona.

\section{DISCUSIÓN}

Es importante tener en cuenta que según la USEPA (15), es más apropiado usar datos de muestras de agua que estimaciones de concentraciones de exposición, cuando los puntos de muestreo corresponden a puntos de exposición (caso de los pozos noria). La USEPA también recomienda precaución al extrapolar los valores de las muestras a otras zonas. Por lo tanto, los mapas elaborados a partir de métodos de interpolación originados en valores de muestras donde el punto de muestreo es el punto de exposición sólo tienen un valor referencial y su utilidad radica en la identificación de zonas hacia donde destinar tiempo y recursos para mejorar y precisar la información disponible.

Los valores de riesgo o de cociente de peligro están sujetos a incertidumbre, es decir, como gran parte de los parámetros utilizados en el cálculo presentan una probabilidad de ocurrencia, el riesgo presenta también

esta característica $(15,16)$. Para tener un mejor conocimiento del modelo de cálculo del cociente de peligro, se realizó un análisis de sensibilidad utilizando la técnica del diagrama de tornado (figura 5). Los factores que contribuyen en mayor medida a la variación del cociente de peligro son, en

\section{FIGURA 5. Diagrama de tornado del cociente de peligro}

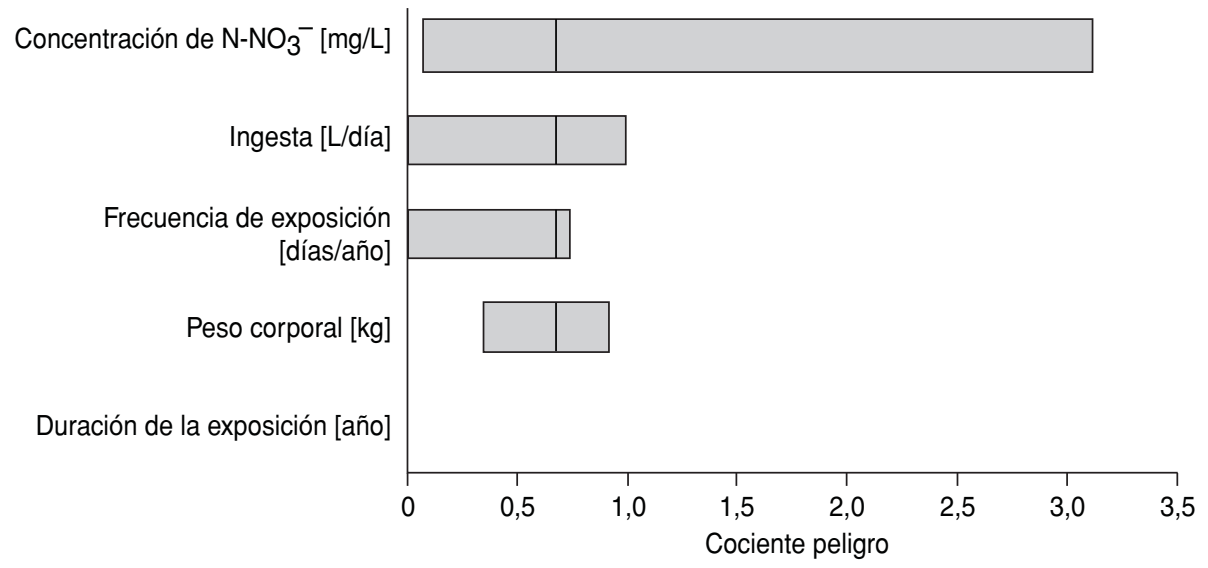


orden de importancia, la concentración de nitrato ( $\mathrm{rs}=0,83)$, la ingesta de agua ( $r s=0,42)$, la frecuencia de exposición ( $\mathrm{rs}=0,4)$ y el peso corporal $(\mathrm{rs}=-0,1)$. Los valores de base de los parámetros determinan un valor del cociente de peligro de 0,69 , lo que significa que en una hipótesis conservadora, en promedio, el coeficiente de peligro del área de estudio se encuentra por debajo del valor límite de seguridad de 1 . De este modo, en general, no existe riesgo para la salud de la población lactante, salvo en casos aislados.
La concentración de nitrato en los pozos de la zona de estudio presenta una distribución espacial que no puede caracterizarse por los métodos tradicionales de variografía. Ello puede deberse a la existencia de factores antrópicos (métodos constructivos o cercanía de animales) que modifican la continuidad espacial del nitrato en el sistema de aguas subterráneas y alteran la calidad del agua de los pozos noria.

En el 14\% de los casos, las concentraciones de nitrato observadas en la zona de estudio se encuentran por encima de las normas chilena e internacionales relativas al agua de bebida. En general, no se espera la aparición de casos de intoxicación en adultos, pero sí existe riesgo para los lactantes alimentados con fórmulas preparadas con agua extraída de estos pozos noria.

Por último, en este tipo de análisis, considerando que no existe continuidad espacial del nitrato en el acuífero y que los puntos de contaminación corresponden a puntos de exposición, no es apropiado hablar de zonas o superficies afectadas, sino que corresponde identificar aquellos pozos que presentan concentraciones de nitrato de riesgo.

\section{REFERENCIAS}

1. Pizarro R, Salazar C, Bravo C, Caro J, Bravo P, Carrasco P. Recursos hídricos. En: Sunkel O, Brzovic F, Romaggi M. Informe País, Estado del Medio Ambiente en Chile-1999. Santiago de Chile: Universidad de Chile, Centro de Análisis de Políticas Públicas; 2000. Pp. 77-129.

2. World Health Organization. Guidelines for Drinking-Water Quality. 2nd ed. Vol 2. Health Criteria and Other Supporting Information. Addendum. Geneva, 1998 (WHO/ EOS/98.1). Hallado en: http://www.who. int/water_sanitation_health/dwq/gdwq2v1/ en/index4.html. Acceso el 16 de agosto de 2006.

3. Toxicity profiles. Toxicity summary for nitrates. [Sitio en Internet] The Risk Asessment Information System (RAIS), Oak Ridge National Laboratory. Hallado en: http://risk. lsd.ornl.gov/tox/profiles/nitrates_f_V1.shtml. Acceso el 16 de agosto de 2006.

4. Fewtrell, L. Drinking-water nitrate, methemoglobinemia, and global burden of disease: a discussion. Environ Health Perspect. 2004; 112(14):1371-74

5. Arumi JL, Oyarzún RA, Sandoval M. A discussion about natural protection again groundwater pollution by nitrates in the Central Valley of Chile. Hydrol Sci J. 2005;50(2): 331-40.

6. Cancino J, Bonilla C, Donoso G. Contaminación de los recursos hídricos en la zona central de Chile. [Sitio en Internet] Actas de la Conferencia Internacional "Los Recursos Hídricos de América Latina en el Umbral del Siglo XXI: Temas Claves para su Desarrollo", VI Jornadas del Comité Chileno para el Programa Hidrológico Internacional (CONAPHI-Chile), Santiago, Chile, mayo de 1999. Hallado en: http:/ / www.unesco.org.uy/phi/libros / VIJornadas/B28.pdf. Acceso el 16 de agosto de 2006

7. Claret M, Ortega M, Mardonez R, Andreu L, Quezada J, Pérez C. Estudio de la contaminación química (fertilizantes) y microbiológica (coliformes) en agua de pozo destinada a consumo humano, del área de influencia del Embalse Digua, Parral, Chile y su dimensión geográfica expresada mediante un SIG. INIA Quilamapu. Chile: Ministerio de Agricultura; Informe Técnico; 2000.

8. Peña CE, Carter DE, Ayala-Fierro F. Toxicología ambiental. Evaluación de riesgos y restauración ambiental. [Sitio en Internet] University of Arizona Superfund Basic Research Program (UA SBRP). Hallado en: http:// superfund.pharmacy.arizona.edu/toxamb/. Acceso el 16 de agosto de 2006.

9. Nuñez J. 2003. Metodología para la evaluación de riesgo asociado a la contaminación de aguas subterráneas [tesis para optar al grado de Magister en Ingeniería Agrícola]. Chillán (Chile): Universidad de Concepción; 2002.

10. Isaaks E, Srivastava R. An Introduction to Applied Geostatistics. New York: Oxford University Press; 1989.

11. United States Environmental Protection Agency. Supplemental Guidance to RAGS: Calculating the concentration term. Intermittent Bulletin, Vol 1, No. 1. Washington: USEPA, 1992. Hallado en: http://www.deq. state.or.us/wmc/tank/documents/epa-ucls. pdf. Acceso el 16 de agosto de 2006.

12. United States Environmental Protection Agency. Integrated Risk Information System (IRIS), Nitrate (CASRN 14797-55-8). Hallado en: http://www.epa.gov/iris/subst/0076. htm. Acceso el 16 de agosto de 2006.
13. Perdomo C, Casanova O, Ciganda V. Contaminación de aguas subterráneas con nitratos y coliformes en el litoral sudoeste del Uruguay. Agrociencia [publicación periódica en línea] 2001. [citada 2006 ago 16]; V(1):[13 pantallas]. Hallado en: http://www.fagro.edu. uy/agrociencia/VOL5/1/P10-22.pdf. Acceso el 16 de agosto de 2006.

14. Cooke SE, Ahmed SM, Macalpine ND. Revised 2005. Introductory Guide to Surface Water Quality Monitoring in Agriculture. Conservation and Development Branch, Alberta Agriculture, Food and Rural Development. Edmonton (Alberta): 2005. Hallado en: http://www1.agric.gov.ab.ca/\$department/ deptdocs.nsf/all/wat2417. Acceso el 16 de agosto de 2006.

15. United States Environmental Protection Agency. Guiding Principles for Monte Carlo Analysis. Washington: USEPA; 1997 (EPA/ 630/R-97/001). Hallado en: http:/ / www.epa. gov/NCEA/pdfs/montcarl.pdf. Acceso el 16 de agosto de 2006.

16. Dawoud E, Purucker S. Quantitative uncertainty analysis of superfund residential risk pathway models for soil and groundwater: White Paper; 1996. [Sitio en Internet] The Risk Asessment Information System (RAIS), Oak Ridge National Laboratory. Hallado en: http: / / rais.ornl.gov/homepage/suprfund. pdf. Acceso el 16 de agosto de 2006.

Manuscrito recibido el 23 de marzo de 2006. Aceptado para publicación, tras revisión, el 4 de septiembre de 2006. 
ABSTRACT Objectives. To assess the risk associated with nitrate contamination of wells that supply drinking water in the rural, Parral region of central Chile.

\section{Risk analysis of nitrate contamination in wells supplying drinking water in a rural area of Chile}

Methods. The nitrate concentration levels were determined using water samples from 94 wells. An analysis of the distribution of nitrate concentration levels was performed in order to assess possible geographic correlations. For the risk analysis, two exposure situations were identified among the population (for adults and for infants), and the health risks were mapped.

Results. Fourteen percent of the wells studied had nitrate concentration levels greater than what the Chilean health standards allow for drinking water. There was no geographic correlation for the nitrate concentration levels. The mean hazard quotient (HQ) for adults in the study area was 0.12, indicating an absence of risk for this population group. For infants, the HQ values had a maximum value of 3.1 in some locations, but the average was 0.69 (still below 1.0), indicating that the well water in the study area was generally not hazardous for infants.

Conclusions. In the Parral region of Chile, nitrate contamination of wells is primarily linked to certain factors such as construction practices and the proximity of livestock. These factors affect the quality of drinking water in isolated cases. There was no risk found for the adult population, but there was for infants fed on formula mixed with water coming from the contaminated wells.

Key words Risk management, water pollutants, nitrates, water purification, rural population, Chile.

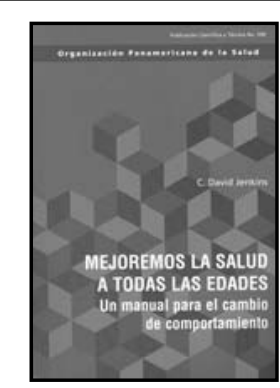

2005, 390pp

ISBN 9275315906 Código: PC 590

Precio: US\$ 30.00 en América Latina y el Caribe/ US\$ 40.00 en el resto del mundo

También disponible en inglés: código SP 590

\section{Mejoremos la salud a todas las edades}

\section{¡Premiado por la British Medical Association en la categoría de salud pública!}

Mejoremos la salud a todas las edades. Un manual para contribuir al cambio del comportamiento es una guía única para ayudar a las comunidades en su lucha por reducir las enfermedades, la discapacidad y la muerte prematura durante todo el ciclo vital. El libro combina estrategias de prevención con principios y métodos prácticos de las ciencias conductuales, siendo por tanto un manual ideal para planificar y poner en práctica programas de salud locales y regionales.

Todo trabajador de salud encontrará en este manual una herramienta esencial. Será especialmente útil en la batalla contra la amenaza creciente de las enfermedades degenerativas crónicas, las enfermedades relacionadas con el modo de vida, la depresión grave, los traumatismos y la violencia.

http://publications.paho.org • Fax: (301) 206-9789 • Correo electrónico:paho@pmds.com 